\title{
DETERMINATION OF CHANGES OCCURING IN CHEMICAL PROPERTIES OF FAT REPEATEDLY USED FOR FOOD FRYING
}

By

Ishara Udeshi Kumari Gunnepana

Degree of Masters of food Science and Technology,

Department of Food Science and Technology, Faculty of Applied Science,

University of Sri Jayawardenapura, Sri Lanka 


\section{DECLARATION}

The work described in tnis thesis were carried by me, under the supervision of Dr. (Mr.) S.B. Nawarathna and the report of this thesis has not been submitted in whole or in part of any University or any other institution for another Degree/Diploma.

Date

I.U.K.Gunnepana 
"I certify that the above statement made by the candidate is true and that this thesis is suitable for submission to the university for the purpose of evaluation"

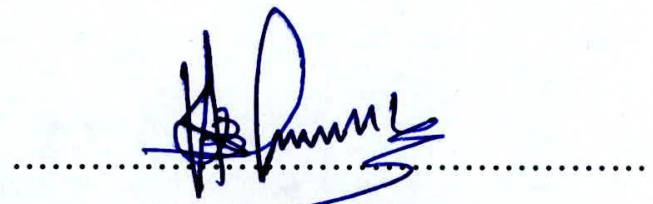

Dr. (Mr.) Dr.S.B. Nawarathna
$12 \cdot 12 \cdot 2015$

Date

Department of Food Science and Technology,

Faculty of Applied Sciences.

University of Sri Jayawardenapura,

Sri Lanka 


\section{TABLE OF CONTENT}

Page

LIST OF CONTENT I

ACKNOLAWDGEMENT III

ABSTRACT IV

Chapter 1- INTRODUCTION

Chapter 2- LITERATURE REVIEW

2.1Fats and Oil 2

2.1.1General Classification 3

2.1.2Chemistry of Fat and Oil Processing 4

2.1.2.1 Refining 4

2.1.2.2 Settling and Degumming 4

2.1.2.3 Neutralization 4

2.1.2.4 Bleaching 5

2.1.2.5 Deodorization 5

2.1.2.6 Hydrogenation 6

2.1.3.1 What is Deep Fat Frying? $\quad 7$

$\begin{array}{lr}\text { 2.1.3.2 Changes in the deep-frying medium } & 8\end{array}$

2.2.1 Coconut oil 9

$\begin{array}{ll}\text { 2.2.2 Palm Oil } & 10\end{array}$

$\begin{array}{ll}\text { 2.2.3 Sunflower Oil } & 13\end{array}$

$\begin{array}{ll}\text { 2.3.1Chemistry of Frying } & 15\end{array}$

2.3.1.1Behavior of the Frying Oil 15

2.3.1.2 Behavior of the Food During Frying 16

$\begin{array}{ll}\text { 2.3.1.3 Chemical and Physical Changes } & 16\end{array}$

2.4 Chemical Properties of an oil 
$\begin{array}{ll}2.4 .3 \text { Saponification value } & 21\end{array}$

2.4.4 Peroxide value $\quad 22$

2.4.5 Gas Chromatography 25

Chapter 3- MATERIALS AND METHOD

3.1 MATERIALS

\subsection{METHODOLOGY}

3.2.1 Preparation of Oil Sample

\subsubsection{Chemical Analysis}

3.2.2.1 Free Fatty Acids content

3.2.2.2 Iodine value

3.2.2.3 Determination of Saponification value 33

3.2.2.4 Determination of Peroxide value 34

3.2.2.5 Determination of fat profile 35

\section{Chapter 4- RESULTS AND DISCUSSION}

4.1 Determination of free fatty acid levels of oils repeatedly used for deep-frying 34

4.2 Determination of Iodine values of oils repeatedly used for deep-frying 37

4.3 Determination of Saponification values of oils repeatedly used for deep-frying 39

4.4 Determination Peroxide values of oils repeatedly used for deep-frying 40

4.5 Determination fat profile of oils repeatedly used for deep-frying 


\section{ACKNOWLEDGMENT}

I express my profound thanks to my honorable supervisor Dr. Nawarathna, senior lecturer, Department of Food Science and Technology, University of Sri Jayawardenapura, for his individual guidance, support and encouragement throughout this research study. And I also grateful to all Academic and non-academic staff for their valuable help and guidance. I must thankful to he staff in Food Laboratory in Sri Lanka Standard Institute, and Chemistry Department in University of Sri Jayawardenapura, who help me in my research work and also Mr.Bandulasoma, Senior Deputy Director and his staff at Industrial Technological Institute for helping me in my research work.

Meantime I must Thank to my beloved parents and family for their unforgettable help, encouragement support and dedication of their several precious hours for my success. 


\title{
DETERMINATION OF CHANGES OCCURING IN CHEMICAL PROPERTIES
}

\author{
OF FAT REPEATEDLY USED FOR FOOD FRYING
}

\begin{abstract}
The Study was done to analyze changes in oil during the frying. The Physical, Chemical properties of oil has changing during heating. There for the quality of oil have been alter. It also results in the production of volatile products such as aldehydes and non volatile fraction which remains in the frying medium. Some of these remaining products have been implicated in producing adverse health effects. Highly oxidized oils may also produce polyaromatic hydrocarbons, which have carcinogenic effect. This work was intended to evaluate the effect of frying on chemical properties of edible oils. The Study was done about three major oils using for deep fat frying in Sri Lanka. Those were Coconut oil, Palm olein and Sunflower oil which are common in Sri Lankan market. These three oils have been use for deep fat frying of meat and potatoes. The samples were subjected to frying with maintained temperature at $200^{\circ} \mathrm{C}$ during frying. Three frying cycles have undertaken for each and oil with meat and potatoes. After frying, the fried oil has been taken for the analysis. The changes in oil characteristics were determined according to the SLS methods. The oil samples were tested for its free fatty acid levels, Iodine value, Saponification value, Peroxide value and to determine Principal constituents of oil. The results have been mention below.
\end{abstract}

The major constituents in coconut oil, palm olein and sunflower oil were lauric acid, palmitic acid and linoleic acid respectively.

The FFA values of coconut oil, palm olein and sunflower oil changed from 0.091 to 0.221 , 0.086 to 0.171 , and 0.060 to 0.121 respectively when meat is fried and changed from 0.091 to $0.234,0.086$ to 0.177 , and 0.060 to 0.101 respectively when potatoes are fried. For coconut oil iodine values have been increased 8.211 to 12.537 when fried meat and 8.211 to 8.494 when fried potato. For palm oil iodine values have been increased 54.207 to 70.234 when fried meat and 54.207 to 75.930 when fried potato, and for sunflower oil iodine values have been increased 106.486 to 122.465 when fried meat and 106.486 to 121.854 when fried potato. For coconut oil saponification values have been increased 252.230 to 267.835 when fried meat and 252.230 to 269.968 when fried potato. For palm oil saponification values have been increased 191.891 to 197.329 when fried meat and 191.891 to 203.597 when fried potato, and for sunflower oil saponification values have been increased 187.194 to 199.644 when fried meat and 187.194 to 197.043 when fried potato. For palm oil peroxide values have been increased 3.466 to 6.172 when fried meat and 3.466 to 6.790 when fried potato, and for sunflower oil peroxide values have been increased 7.556 to 9.993 when fried meat and 7.556 to 9.973 when fried potato. When the results have compared with Sri Lankan Standards requirements, Sunflower oil showed much stability than coconut oil and palm oil through out the frying process. The formation of free fatty acid was low in sunflower oil. And also the degrees of unsaturation were within the limits. Therefore, the above analyses have confirmed that the sunflower oil is more stability and more suitable for repeated deepfrying. (Key words: Coconut oil, Palm olein, Sunflower oil, Free fatty acids, Iodine value, Saponification value, Peroxide value) 


\section{INTRODUCTION}

\section{Chapter-1}

In our country, different oils are used in frying purpose. Ex: Coconut oil, Palm oil, Sunflower oil, Olive oil...etc. With the increasing temperature of oil the physical, chemical and organoleptic properties are changing. These changing properties can create adverse effects on our health.

During the frying process, oil undergoes changes in physical, chemical, nutritional and sensory properties, which affects its frying performance. The repeated use of oil could affect the shelf life and nutritional quality of fried foods due to the development of rancidity in the frying oil taken up by the products. High frying temperature, associated with the presence of air and moisture, lead to production of breakdown products that include volatile and non-volatile compounds. These products result from oxidation of unsaturated fatty acids, lipid hydrolysis, and transformation of linear fatty acids in cyclical compounds and fatty acid or lipid polymerization. The intensity of these reactions depends on duration, method of heat treatment, frying medium and type of product.

Therefore, in order to protect the health of the public, it is essential not only to monitor the quality of oils used, but also to determine their stability under normal conditions of use in cooking. The frying operations were applied on meat and potatoes which are common products prepared by deep frying in Sri Lanka. So it is important to identify how these changes happening between different oil varieties and to choose best oil for frying among those selected oil varieties.

The general objective of this study was to evaluate the effect of frying on chemical properties of three brands of oils in Sri Lankan market in order to assess whether these oils could be re-used or not. Other specific objectives are to determine the changes of free fatty acids, Peroxide value, iodine value, saponification value, and fatty acid composition of oil repeatedly used for frying. 


\section{Chapter-2}

\section{LITERATURE REVIEW}

\subsection{Fats and Oil}

"Like most organic materials, Oils and fats are made up of three elements:-

1. Carbon

2. Oxygen

3. Hydrogen

These elements combine together to form chains known as fatty acids. The tryglyceride molecule is the basis of all oils and fats. Three of these chains then join together to form a molecule known as a triglyceride." ( http://www.neoda.org.uk/oils-fats-information)

"Oils and fats vary in both their appearance and functionality due to differences in the types of fatty acid chain which join together to form the triglyceride molecule.

Individual fatty acids can be either saturated or unsaturated and the latter being further subdivided into mono and polyunsaturated. All oils contain a proportion of all three types and it is this combination in any oil, which has been determine whether it will be solid fat or liquid oil at ambient temperature. The functionality of liquid oils and solid fats varies considerably and which to choose will depend on the intended application.

Generally speaking, oils containing a greater proportion of unsaturated fatty acids are liquid at room temperature whereas those with higher amounts of saturated fatty acids will be solid.” ( http://www.neoda.org.uk/oils-fats-information)

\section{DEFINITION OF FATS AND OIL}

"It represent the most prevalent single category of a series of compounds known as lipids. The word "lipide" is defined in webster's unabridged disitionary as, any of a group of substances that in general are soluble in eather, chloroform, or other solvents for fats but are only sparingly soluble in water, that with proteins and carbohydrates constitute the 
principal structural components of living cell, and related and derived compounds, and some times steroids and carotenoids" (Dugan, 1996)

\subsubsection{General Classification}

"Simple Lipids (neutral lipids) - Esters of fatty acids with alcohols.

Fats: Esters of fatty acids with glycerol

Waxes: Esters of fatty acids with alcohols other than glycerol

Compound lipids - Compounds containing other groups in addition to an ester of a fatty acid with an alcohol

Phospholipids (phosphatides): esters containing fatty acids, phosphoric acid, and other groups usually containing nitrogen

Cerebrocides(glycolipids): compounds containing fatty acids, a carbohydrate and nitrogen moiety, but no phosphoric acid

Other compound lipids- sphingolipids and sulpholipids

Derived lipids- Substances derived from neutral lipids or compound lipids and having general properties of lipids

Fatty acids

Alcohols: usually normal chain higher alcohols and sterols

Hydrocarbons

Fats and oils are the most concentrated source of energy. They provide $9 \mathrm{kcal}$ of energy per gram which is approximately double the energy provided by proteins or carbohydrates. They are carriers of fat-soluble vitamins, and they contribute to food flavor and palatability as well as to the feeling of satiety after eating." (Dugan,1996) 


\subsubsection{Chemistry of Fat and Oil Processing}

\section{"2.1.2.1 Refining}

Crude oils and fats contain varying amounts of substances that may impart undesirable flavor, color, or keeping quality. These substances were included free fatty acids, phospholipids, carbohydrates, proteins and their degradation products, water, pigments mainly carotenoids and chlorophyll, and fat oxidation products. Crude oils have been subject to several commercial refining processes designed to remove these materials.

\subsubsection{Settling and Degumming}

Settling have been involve heating the fat and allowing it to stand until the aqueous phase separates and can be withdrawn. This rids the fat of water, proteinaceous material, phospholipids, and carbohydrates. In some cases, particularly with oils containing substantial amounts of phospholipids (e.g., soybean oil), a preliminary treatment known as degumming is applied by adding $2-3 \%$ water, agitating the mixture at about $50^{\circ} \mathrm{C}$, and separating the hydrated phospholipids by settling or centrifugation.

\subsubsection{Neutralization}

To remove free fatty acids, caustic soda in the appropriate amounts and strength were mix with the heated fat and the mixture was allowed to stand until the aqueous phase settles. The resulting aqueous solution, called foots or soap stock, is separated and used for making soap. Residual soap stock has been removed from the neutral oil by washing it with hot water, followed by settling or centrifugation.

Although free fatty acid removal is the main purpose of the alkali treatment, this process also results in a significant reduction of phospholipids and coloring matter. (Nawar,2008) 


\subsubsection{Bleaching}

An almost complete removal of coloring materials can be accomplished by heating the oil to about $85^{\circ} \mathrm{C}$ and treating it with adsorbants, such as fuller's earth or activated carbon. Precautions should be taken to avoid oxidation during bleaching. Other materials, such as phospholipids, soaps, and some oxidation products, are also absorbed along with the pigments. The bleaching earth is then removed by filtration.

\subsubsection{Deodorization}

Volatile compounds with undesirable flavors, mostly arising from oxidation of the oil, were removing by steam distillation under reduced pressure. Citric acid was often added to sequester traces of pro-oxidant metals. It is believed that this treatment also results in thermal destruction of nonvolatile off-flavor substances, and that the resulting volatiles are distilled away.

Although the oxidative stability of oils is generally improved by refining, this is not always the case. Crude cottonseed oil, for example, has a greater resistance to oxidation than its refined counterparts due to the greater amounts of gossypol and tocopherols in the crude oil. On the other hand, there can be little doubt as to the remarkable quality benefits that accrue from refining edible oils. An impressive example is the upgrading of palm oil quality that has occurred in recent years. Furthermore, in addition to the obvious improvements in color, flavor, and stability, powerful toxicants (e.g., aflatoxins in peanut oil and gossypol in cottonseed oil) are effectively eliminated during the refining process.

(Nawar,2008) 


\subsubsection{Hydrogenation}

Hydrogenation of fats involves the addition of hydrogen to double bonds in the fatty acid chains. The process is of major importance in the fats and oils industry. It accomplishes two major objectives. First, it allows the conversion of liquid oils into semisolid or plastic fats more suitable for specific applications, such as in shortenings and margarine, and second, it improves the oxidative stability of the oil.

In practice, the oil is first mixed with a suitable catalyst (usually nickel), heated to the desired temperature $\left(140-225^{\circ} \mathrm{C}\right)$, then exposed, while stirred, to hydrogen at pressures up to 60 psig. Agitation is necessary to aid in dissolving the hydrogen, to achieve uniform mixing of the catalyst with oil, and to help dissipate the heat of the reaction. The starting oil must be refined, bleached, low in soap, and dry; the hydrogen gas must be dry and free of sulfur, $\mathrm{CO} 2$, or ammonia; and the catalyst must exhibit long-term activity, function in the desired manner with respect to selectivity of hydrogenation and isomer formation, and be easily removable by filtration.

The course of the hydrogenation reaction is usually monitored by determining the change in refractive index, which is related to the degree of saturation of the oil. When the desired end point is reached, the hydrogenated oil is cooled and the catalyst is removed by filtration." (Nawar, 2008) 


\subsubsection{What is Deep Fat Frying?}

"Deep-frying is a cooking process, with which water containing foodstuff is immersed into edible oils or fats at temperatures between $140-180^{\circ} \mathrm{C}$. In the first phase, within a few seconds, a thin crust forms, whose structure crucially affects the deep-frying process and the quality of the food with regards to fat absorption and crispness.

Fats and oils have a high heat capacity, thereby enabling heat transfer at temperatures far above that of the boiling point of water. Due to the evaporation in the boundary zone between food and oil, the water bound in the food is gradually transported from the inside to the boundary layer into the surrounding oil (mass transfer). The speed of transfer depends more or less on the structure of the outer crust. As soon as the transfer of water ends, the temperature inside the food starts to rise above $100^{\circ} \mathrm{C}$. At this point the typical deep-frying aromas and flavors as well as the gold-yellow colour begin to develop. With the rise of temperature in the boundary zone to more than $120^{\circ} \mathrm{C}$, the formation of acrylamide begins, in particular in the presence of reducing sugars and asparagine like in grain- or potato products.

The moisture released from the food acts as a protective shield, preventing direct contact of oxygen to the fat surface. Consequently, frying fats that are constantly used for the preparation of meals deteriorate more slowly as if being heated up frequently without food." (Schwarzinger., et al,2006) 


\subsubsection{Changes in the deep-frying medium}

"Depending upon temperature and the duration of the deep-frying process, the heating of fats and oils will change the composition of the medium and eventually lead to the degradation of the fat. In order to produce optimal sensory results the oil must have experienced some initial thermal decomposition.

On the other hand fat degradation is not reversible, the processing must aim at obtaining and maintaining optimal conditions for the production of tasty food for as long a time as possible. This is not a simple task, as complex physical procedures and chemical reactions take place during deep frying, which are influenced by temperature and also by the interaction between the oil/fat, the food item and atmospheric oxygen.

\section{Decomposition:}

Food fats and oils are (from a chemical point of view) mixtures of triacylglycerols (nonpolar components), which are composed of fatty acids and glycerol. Such triacylglycerols are affected by oxygen and heat, whereby, due to oxidation and polymerization more polar compounds like free short-chain fatty acids, mono- and diglycerides, aldehydes, ketones, polymers, cyclic and aromatic compounds are formed. Some of these compounds are responsible for the pleasant flavour of the deep-fried products. At the same time however, oxidation products such as short-chain fatty acids lead to a decrease of the smoke point, so that the fat starts smoking at clearly lower temperatures than the fresh fat. In addition it develops a gritty taste. Polymeric components lead to the formation of foam and increase the viscosity (i.e. the deep-frying medium becomes more viscous). Especially when palm kernel oil and coconut fat are used, water from the fried food splits off fatty acids (hydrolysis), forming an eye-irritating smoke due to the formation of toxic acrolein and free short and middle chained fatty acids. With other fats and oils the hydrolytic splitting rises only to a very small extent." (Schwarzinger., et $a l, 2006)$ 